\title{
Uma abordagem neural na identificação de objetos em imagens para auxilio na manutenção de rede elétrica
}

\author{
Regis Yuri Olivo \\ Universidade Tecnológica Federal do Paraná \\ Medianeira, Paraná, Brasil \\ Email: regisolivo at alunos dot utfpr dot edu dot br \\ Arnaldo Candido Junior \\ Universidade Tecnológica Federal do Paraná \\ Medianeira, Paraná, Brasil \\ Email: arnaldoc at utfpr dot edu dot br
}

\author{
Pedro Luiz de Paula Filho \\ Universidade Tecnológica Federal do Paraná \\ Medianeira, Paraná, Brasil \\ Email: pedrol at utfpr dot edu dot br
}

\begin{abstract}
Resumo-Problemas causados pelas redes de distribuição de energia elétrica podem gerar grandes prejuízos a empresas e pessoas, com perdas de matéria prima, danos em equipamentos ou até descarga elétrica podendo levar um individuo à óbito. Com o objetivo de ajudar nas inspeções para manutenção desses componentes que são feitas manualmente, este projeto propõem uma abordagem neural na identificação de objetos em imagens capturadas por drones para auxiliar na manutenção das redes elétrica. Para tanto, foi feito o treinamento de uma rede neural artificial (RNA) Darknet-53 do YOLOv3 no framework Tensorflow, permitindo a classificação e detecção, em tempo real, de componentes presentes nas redes de distribuição de energia. Foram definidas sete classes de objetos, e obteve-se $84,16 \%$ de precisão de variação média (mAP).
\end{abstract}

Palavras-chave: aprendizagem profunda, redes neurais, detecção de objetos.

\begin{abstract}
Electricity distribution networks are susceptible to problems that can generate great losses to companies and people, including loss of raw material, damage to equipment or even life threatening electrical discharges. In order to assist in the inspections for maintenance of these components that are done manually, this project proposes a neural approach in the identification of components of the electrical network in images captured by drones to assist in the maintenance of the electrical networks. The artificial neural network Darknet-53 was trained for this task, allowing real time component detection and classification. Seven classes of electricity distribution related objects were defined, and $84.16 \%$ accuracy of mean variation (mAP) was obtained.
\end{abstract}

Keywords: deep learning, neural networks, object detection.

\section{INTRODUÇÃo}

A energia elétrica é uma das matérias primas mais essenciais em uma sociedade, sendo ela cada vez mais utilizada, principalmente por indústrias. Como a eletricidade é vendida como um produto, tornou-se necessário estabelecer alguns parâmetros em suas características de qualidade para garantir que seu fornecimento seguisse dentro dos parâmetros estabelecidos [1].

Problemas que se referem a qualidade da eletricidade são de forma geral, defeitos em equipamentos que alimentam a rede de distribuição de energia elétrica. Esses defeitos nos equipamentos que não são solucionados de forma adequada, podem causar danos em máquinas como a redução da vida útil e até mal estar em pessoas como perturbações causadas por ressonâncias eletromagnéticas [2].

Para diminuir tais problemas, seria interessante métodos preventivos, que evitem/minimizem os defeitos apresentados pelas redes de distribuição de energia. Nesse sentido, o uso de Visão Computacional pode auxiliar. Na visão computacional alguns elementos são importantes, como a captura das imagens e o processamento de seus dados. Para esse processamento eficiente desses dados, as Redes Neurais Artificiais (RNAs) são muito úteis.

As RNAs são ferramentas de Inteligência Artificial (IA) desenvolvidas que se baseiam nos neurônios humanos. Elas são aplicadas em diversas tarefas, como mineração de dados, aprendizado de máquina para classificação, regressão entre outras [3].

Existem alguns trabalhos na identificação de problemas na rede elétrica. Um desses trabalhos é o apresentado em [4], que identifica problemas em espaçadores nas linhas de transmissão de energia utilizando RNAs. O objetivo foi automatizar as inspeções do estado dos espaçadores nas linhas de transmissão, para isso a RNA foi treinada para predizer falhas no funcionamento dos mesmos. A rede neural foi submetida a um teste com 20 imagens, com 11 imagens classificadas em "bom estado" e 9 imagens classificadas "com necessidade de manutenção". Apenas 3 imagens foram classificadas de forma incorreta, sendo 2 falsos positivos e 1 falso negativo.

Em [5], são propostos alguns métodos para classificar falhas nos equipamentos da rede elétrica causados por variações de 
calor. Nos métodos propostos, são utilizados algoritmos de IA para tomada de decisão, a partir de bases de imagens térmicas. Todos os métodos utilizados obtiveram uma acurácia entre $65 \%$ e $80 \%$.

A proposta de [6], faz o treinamento de uma RNA para obter diagnóstico de transformadores imersos em líquido isolante. Os dados de entrada são obtidos por meio de análise cromatográfica e são relacionados a concentração de três gases dentro do transformador e a proporção entre essas concentrações. Os autores chegaram a taxas de acerto superiores a $95 \%$ de acordo com a topologia neural utilizada.

Os exemplos apresentados, demonstram o avanço das RNAs e importância que as mesmas vem ganhando na melhoria na qualidade nas redes elétricas. Com o intuito de colaborar com isso, o objetivo deste projeto foi treinar uma RNA para classificação e detecção dos componentes presentes nas redes elétricas a partir de imagens obtidas em campo.

\section{Metodologia}

Como não existem bases públicas disponíveis para o objetivo proposto, uma base de imagens foi criada especificamente para o treinamento da rede neural. Em conjunto com o Parque Tecnológico de Itaipu (PTI), foram realizadas coletas das imagens na região de Foz do Iguaçu - PR, com o auxilio de um drone. As imagens foram adquiridas em distintas coletas, e variam a sua resolução de $4.000 \times 2.250$ pixeis até 5.472 $\times 3.078$ pixeis. A base final ficou composta por 824 imagens, nas quais encontram-se objetos como transformadores, espaçadores, para-raios, isoladores de porcelana, isoladores de suspensão, fusíveis e cobertura GL (Grampo de Linha). A Figura 1, apresenta exemplos desses objetos.

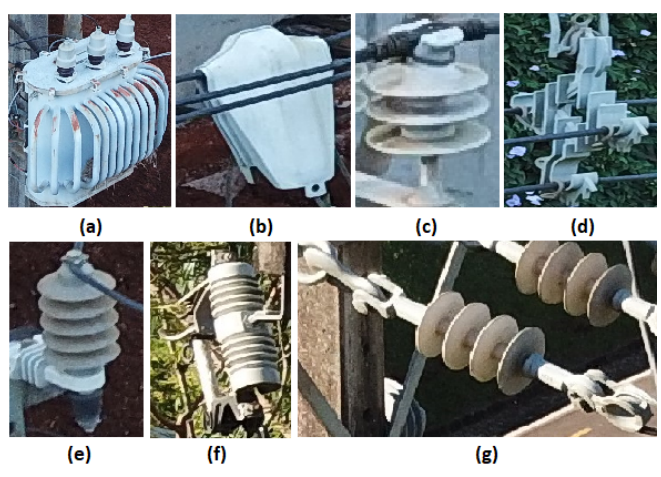

Fig. 1. Transformador (a), Cobertura GL (b), Isolador de Porcelana (c), espaçador (d), para-raio (e), Fusível (f) e Isolador de Suspensão (g).

Com intuito de aumentar a quantidade de imagens, e por consequência, melhorar o treinamento da RNA, algumas imagens foram divididas em uma ou mais vezes, totalizando ao final um total de 1.268 imagens contidas na base final.

Como se trata de um problema de detecção de objetos, foi necessário rotular toda base de dados no formato pascalVOC $^{1}$, utilizando uma ferramenta de anotação gráfica de

\footnotetext{
${ }^{1}$ https://pjreddie.com/projects/pascal-voc-dataset-mirror
}

código aberto chamada LabelImg ${ }^{2}$, através da qual foram rotulados um total de 492 transformadores, 1.611 isoladores de porcelana, 681 isoladores de suspensão, 1.173 fusíveis, 828 para-raios, 390 espaçadores e 491 coberturas GL.

Para o treinamento da rede, foram divididas 293 imagens da base para o conjunto de teste e 975 imagens para o conjunto de treino e validação. A Tabela I mostra a quantidade de objetos de cada classe separados para conjunto de teste e conjunto de treino e validação.

TABELA I

QUANTIDADE DE OBJETOS DO CONJUNTO DE TESTE E CONJUNTO DE TREINO E VALIDAÇÃO

\begin{tabular}{|c|c|c|}
\hline Objeto & Teste & Treino e Validação \\
\hline Transformador & 122 & 370 \\
\hline Isolador de porcelana & 404 & 1207 \\
\hline Isolador de suspensão & 189 & 492 \\
\hline Fusível & 289 & 884 \\
\hline Para-raio & 213 & 615 \\
\hline Espaçador & 74 & 316 \\
\hline Cobertura GL & 130 & 361 \\
\hline
\end{tabular}

O framework utilizado para detecção de objetos foi o Tensorflow, uma biblioteca de código aberto para criação e implementação de modelos de Aprendizagem de Máquina. Esse framework pode ser usado para vários fins, como aplicações em reconhecimento de fala, robótica, visão computacional, entre outros [7].

Como o objetivo do projeto é classificar e detectar objetos em tempo real com o auxílio de drones na inspeção preventiva de elementos da rede de distribuição de energia, com o propósito de aumentar sua confiabilidade, foi escolhido o uso da Rede Neural Artificial Darknet-53 do YOLOv3, que atende os objetivos para classificar e detectar os objetos propostos no projeto.

Na Figura 2 é apresentado a arquitetura da rede, formada por 53 camadas convolucionais e acompanhada pela função softmax para normalizar a saída na última camada.

No treinamento da rede foi usada uma máquina com processador Intel Core i7-8700 de 3,20 GHZ, Memória RAM de 16 GB DDR4 e Placa de video NVIDIA Titan V com 12 GB HBM2 5120 CUDA Cores. O treinamento durou cerca de duas semanas, com aproximadamente 310.000 iterações.

As métricas de avaliação utilizadas foram: Intersecção sobre União $(\mathrm{IoU}) \geq 0.5$ e Precisão de Variação Média (mAP).

A IoU é uma das métricas mais populares utilizadas em RNAs de detecção de objetos. Seu cálculo mede a sobreposição entre 2 limites $A, B \subset \mathbb{S} \in \mathbb{R}^{n}$. A IoU é apresentada na Equação 1 [8]. A Figura 3, apresenta uma representação visual desta métrica.

$$
I o U=\frac{|A \cap B|}{|A \cup B|}
$$

\footnotetext{
${ }^{2}$ https://github.com/tzutalin/labelImg
} 


\begin{tabular}{|c|c|c|c|c|}
\hline & Тіро & Filtros & Tamanho & Saída \\
\hline & Convolucional & 32 & $3 \times 3$ & $256 \times 256$ \\
\hline & Convolucional & 64 & $3 \times 3 / 2$ & $128 \times 128$ \\
\hline \multirow{5}{*}{$1 \mathrm{X}$} & Convolucional & 32 & $1 \times 1$ & \\
\hline & Convolucional & 64 & $3 \times 3$ & \\
\hline & Residual & & & $128 \times 128$ \\
\hline & Convolucional & 128 & $3 \times 3 / 2$ & $64 \times 64$ \\
\hline & Convolucional & 64 & $1 \times 1$ & \\
\hline \multirow[t]{4}{*}{$2 \mathrm{X}$} & Convolucional & 128 & $3 \times 3$ & \\
\hline & Residual & & & $64 \times 64$ \\
\hline & Convolucional & 256 & $3 \times 3 / 2$ & $32 \times 32$ \\
\hline & Convolucional & 128 & $1 \times 1$ & \\
\hline \multirow[t]{4}{*}{$8 \mathrm{X}$} & Convolucional & 256 & $3 \times 3$ & \\
\hline & Residual & & & $32 \times 32$ \\
\hline & Convolucional & 512 & $3 \times 3 / 2$ & $16 \times 16$ \\
\hline & Convolucional & 256 & $1 \times 1$ & \\
\hline \multirow[t]{4}{*}{$8 X$} & Convolucional & 512 & $3 \times 3$ & \\
\hline & Residual & & & $16 \times 16$ \\
\hline & Convolucional & 1024 & $3 \times 3 / 2$ & $8 \times 8$ \\
\hline & Convolucional & 512 & $1 \times 1$ & \\
\hline \multirow[t]{3}{*}{$4 \mathrm{X}$} & Convolucional & 1024 & $3 \times 3$ & \\
\hline & Residual & & & $8 \times 8$ \\
\hline & $\begin{array}{l}\text { Pool Médio } \\
\text { Conectado } \\
\text { Softmax }\end{array}$ & & \multicolumn{2}{|l|}{1000} \\
\hline
\end{tabular}

Fig. 2. Arquitetura da rede neural Darknet-53 do YOLOv3.

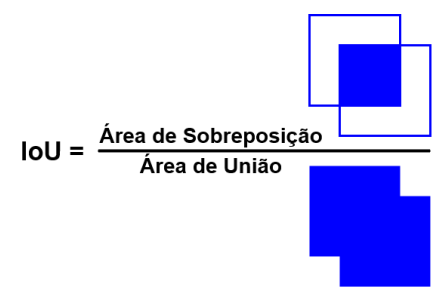

Fig. 3. Métrica de Intersecção sobre União (IoU) Adaptado de [8].

A métrica mAP também é usada para medir a qualidade da detecção de objetos [9]. Essa métrica é baseada em outras duas, a Precisão (P) e Revocação ou Recall (R), apresentadas nas equações 2 e 3 , no qual TP, FP e FN representam verdadeiro positivo, falso positivo e falso negativo respectivamente [10].

$$
\begin{aligned}
& P=\frac{T P}{T P+F P} \\
& R=\frac{T P}{T P+F N}
\end{aligned}
$$

Usando os valores de $\mathrm{P}$ e $\mathrm{R}$, é construído um gráfico de eixos $X$ e $Y$, no qual o eixo $X$ representa os valores de $\mathrm{R}$ e o eixo $Y$ representa os valores de $\mathrm{P}[10]$.

Para achar o valor de AP é usado a equação 4.

$$
A P=\sum_{n}\left(R_{n}-R_{n-1}\right) P(n)
$$

Os valores de AP variam de 0 a 1 , em que os valores $P_{n}$ e $R_{n}$ correspondem a Precisão e Recall na enésima limiar. Com isso, é possível achar o mAP, que corresponde a média dos valores de AP de todas as classes de objetos.

\section{Resultados E Discussões}

Os resultados obtidos no projeto mostram que mesmo com uma base de imagens muito pequena em comparação as bases de imagens publicamente disponíveis para detecção de objetos, é possível treinar uma rede neural artificial para a tarefa. $\mathrm{Na}$ Figura 4 apresenta-se a precisão de variação média obtida.

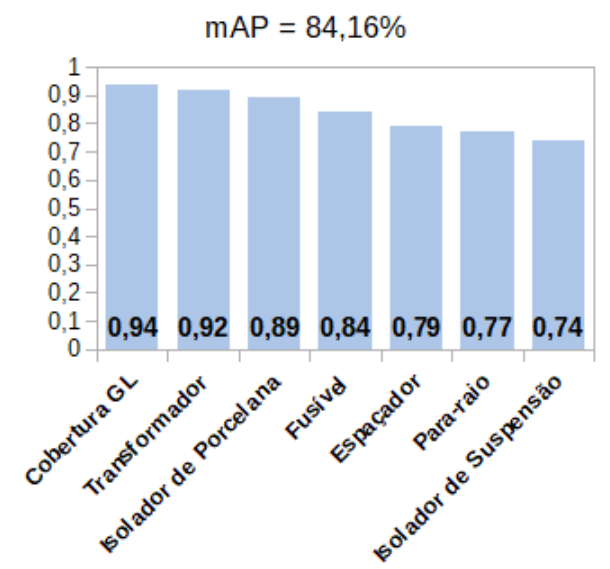

Fig. 4. Resultado de AP do Conjunto de Teste.

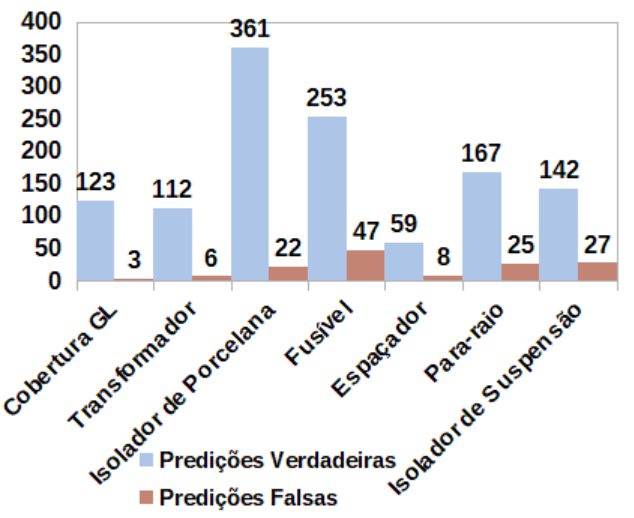

Fig. 5. Resultado das predições do conjunto de teste.

Analisando a Figura 4, é possível determinar que a Cobertura GL e Transformador tiveram um AP maior que 0.90, com 0.94 e 0.92 respectivamente. Já o isolador de suspensão, pararaio e espaçador foram os piores resultados, com um AP de $0.74,0.77$ e 0.79 respectivamente, únicos valores menores que 0.80 . A quantidade de espaçadores no conjunto de teste foi o menor entre todos, com base nisso é possível determinar que seu resultado abaixo de $0.80 \mathrm{se}$ deu por conta da pequena quantidade de objetos dessa classe no conjunto de treinamento. O mAP obtido foi $84.16 \%$, mesmo usando uma base de imagens pequena.

Na Figura 5, são apresentadas as predições de todos os objetos, na qual a Cobertura GL e o Transformador obtiveram predições falsas de apenas 3 e 6 , respectivamente. A classe 
que mais obteve predições falsas, em valores absolutos, foi o fusível, com 47 erros.

As Figuras 6 e 7, mostram a detecção de alguns dos objetos.

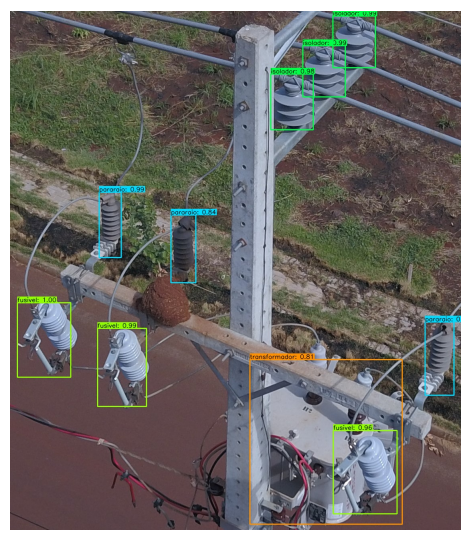

Fig. 6. Detecção dos objetos.

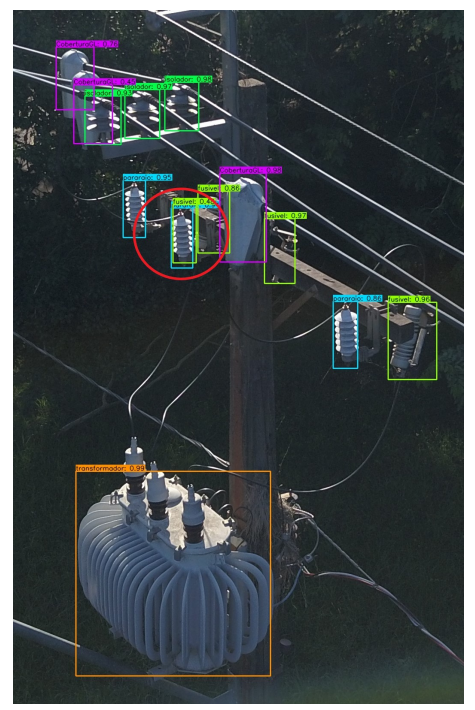

Fig. 7. Detecção dos objetos.

Na Figura 6 é observado que foram detectados três fusíveis, três para raios, três isoladores de porcelana e um transformador, todos detectado corretamente. Na Figura 7 foram detectados um transformador, três coberturas GL, três para raios, três isoladores de porcelana e quatro fusíveis, porém um dos fusíveis (destaque em vermelho) foi detectado erroneamente em cima de um para raio.

\section{CONSIDERAÇÕES FINAIS}

Neste artigo foi apresentada a construção de uma base de imagens por meio de drones e de um modelo de detecção de objetos baseado em redes neurais para análise de sete componentes comuns em linhas de transmissão de eletricidade.

Os resultados obtidos mostram que, mesmo com uma base de imagens pequena, e não balanceada, é possível treinar uma rede neural artificial para a detecção das classes de objetos elétricos estudadas. Um ponto importante é que a base de imagens utilizada neste projeto para o treinamento da rede neural, continha objetos com diferentes modelos, cores e tamanhos, e ainda assim foi possível obter-se um mAP $84,16 \%$, com destaque para transformadores e coberturas GL que obtiveram AP superiores a 90\%. Observa-se ainda que alguns objetos como o transformador continham cerca de 4 modelos diferentes. Na sequencia do projeto, serão capturadas novas imagens e usadas técnicas de data augmentation, com intuito de buscar um maior balanceamento entre classes.

\section{AGRADECIMENTOS}

Um agradecimento a Fundação Parque Tecnológico Itaipu (FPTI) pela criação do dataset com imagens de alta resolução, ao Centro de inteligência e Gestão Territorial (IT.DT) por propor o projeto, ao Centro de Tecnologias Abertas e IoT(TA.DT) pelo financiamento do projeto, a Itaipu pelo incentivo ao projeto e também a NVIDIA pela doação da placa de video TITAN V utilizada no treinamento da RNA.

\section{REFERÊNCIAS}

[1] E. Martinho, Distúrbios da Energia Elétrica. Saraiva Educação S.A., 2009. [Online]. Available: https://books.google.com.br/books?id= CYywDwAAQBAJ

[2] S. M. Deckmann and J. A. Pomilio, Avaliação da Qualidade da Energia Elétrica. Departamento de Sistemas e Energia (DSE), 2018. [Online]. Available: http://www.dsce.fee.unicamp.br/ antenor/pdffiles/ qualidade/a1.pdf

[3] A. R. S. Enriquez, "Diagnóstico de falhas em transformadores de potência através de análise de gases dissolvidos usando rede neural artificial," Master's thesis, Universidade Federal do Maranhão, Centro de Ciências Exatas e Tecnologia, Programa de Pós-Graduação em Engenharia Elétrica, São Luís, 2020.

[4] E. Oroski, "Identificação de falhas em espaçadores de linhas de transmissão utilizando visão estéreo e redes neurais artificiais," Master's thesis, Universidade de Brasília, Faculdade de Tecnologia, Departamento de Engenharia Elétrica, Brasília, 2011.

[5] M. O. Yuri, "Detecção automática de problemas elétricos por meio da análise computacional de imagens térmicas," Master's thesis, Centro Universitário FEI, Programa de Pós-Graduação Stricto Sensu em Engenharia Elétrica, São Bernardo do Campo, 2019.

[6] M. Mendonça, M. A. F. Finocchio, and L. F. M. Gonçalves. (2020, Jul.) Redes neurais artificiais aplicadas no auxílio de diagnóstico de falhas em transformadores.

[7] M. Abadi, A. Agarwal, P. Barham, E. Brevdo, Z. Chen, C. Citro, G. S. Corrado, A. Davis, J. Dean, M. Devin, S. Ghemawat, I. J. Goodfellow, A. Harp, G. Irving, M. Isard, Y. Jia, R. Józefowicz, L. Kaiser, M. Kudlur, J. Levenberg, D. Mané, R. Monga, S. Moore, D. G. Murray, C. Olah, M. Schuster, J. Shlens, B. Steiner, I. Sutskever, K. Talwar, P. A. Tucker, V. Vanhoucke, V. Vasudevan, F. B. Viégas, O. Vinyals, P. Warden, M. Wattenberg, M. Wicke, Y. Yu, and $\mathrm{X}$. Zheng, "Tensorflow: Large-scale machine learning on heterogeneous distributed systems," CoRR, vol. abs/1603.04467, 2016. [Online]. Available: http://arxiv.org/abs/1603.04467

[8] S. H. Rezatofighi, N. Tsoi, J. Gwak, A. Sadeghian, I. D. Reid, and S. Savarese, "Generalized intersection over union: A metric and A loss for bounding box regression," CoRR, vol. abs/1902.09630, 2019. [Online]. Available: http://arxiv.org/abs/1902.09630

[9] J. Redmon, S. K. Divvala, R. B. Girshick, and A. Farhadi, "You only look once: Unified, real-time object detection," CoRR, vol. abs/1506.02640, 2015. [Online]. Available: http://arxiv.org/abs/1506. 02640

[10] G. A. d. L. Freitas, "Aprendizagem profunda aplicada ao futebol derobôs: Uso de redes neurais convolucionais paradetecção de objetos," Master's thesis, Universidade Estadual de Londrina, Universidade Estadual de LondrinaCentro de Tecnologia e Urbanismo, Departamento de Engenharia Elétrica, Londrina, 2019. 